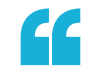

a spatiotemporal
distinction

between antigen

uptake in the

alveoli and

antigen retention

and presentation

in the airway

regions that are

adjacent to the

alveoli

\title{
ANTIGEN PRESENTATION
}

\section{Visualizing DC dynamics in the lung}

In this study, Krummel and colleagues used two-photon microscopy to visualize the dynamics of antigen uptake and presentation by dendritic cells (DCs) in the lung and identified a spatiotemporal distinction between antigen uptake in the alveoli and antigen retention and presentation in the airway regions that are adjacent to the alveoli.

In the trachea, DCs have been shown to project dendrites into the airspace and take up antigens in response to Toll-like receptor 4 (TLR4) stimulation, but technical limitations have restricted the visualization of immune cell interactions in the lung. Recent advances allowed the authors of this study to apply two-photon imaging to viable lung slices and intravital stabilized lung parenchyma, and thus to examine DC dynamics deeper in the lung. Cd11c-EYFP (enhanced yellow fluorescent protein) mice - which allow for the visualization of DCs and macrophages - were subjected

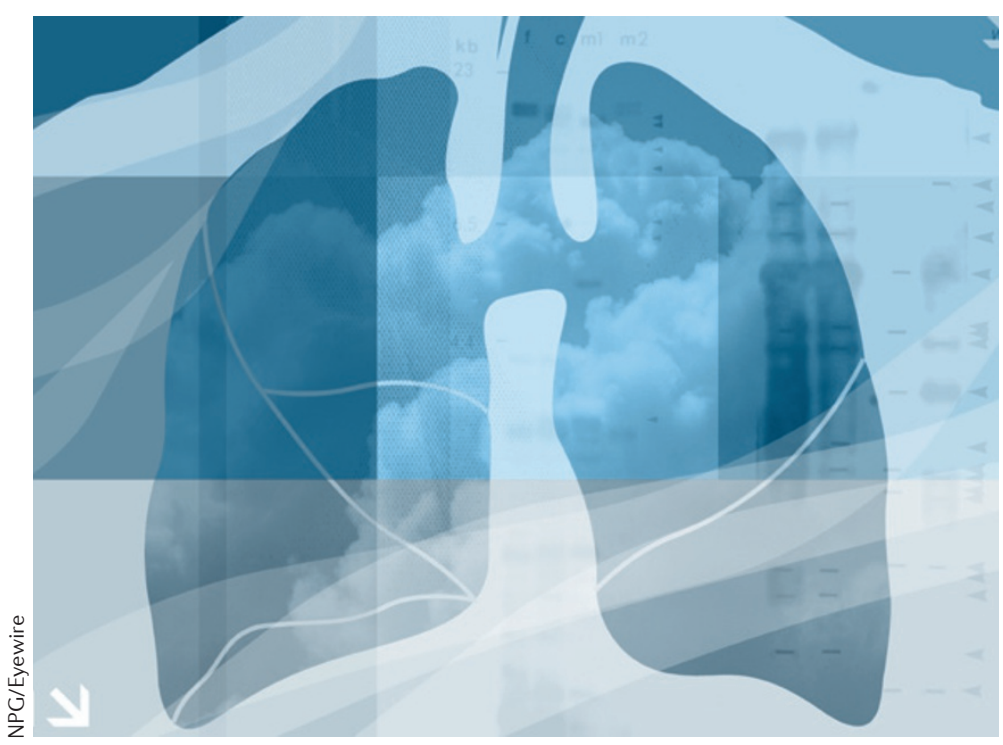

to the commonly used ovalbumin and alum model of allergic airway inflammation.

$\mathrm{EYFP}^{+}$cells were found to accumulate near the allergic airways, and further analysis identified these cells as CD11b+ DCs. These airwayadjacent DCs were highly motile, and this behaviour was not altered by allergen challenge. Only $3 \%$ of airway-adjacent DCs generated dendrite processes, and these processes did not extend into the airway lumen. Furthermore, process formation did not change in response to allergen challenge or following administration of the TLR4 ligand lipopolysaccharide.

By contrast, alveolar macrophages and DCs displayed very little motility. Furthermore, almost all alveolar DCs had active dendrites in both the steady state and following allergen challenge. These dendrites moved along the mucosal face of alveolar epithelial cells. Furthermore, when ovalbumin-sensitized mice were administered microbeads and ovalbumin intranasally, within 1 hour alveolar DCs, but not airwayadjacent DCs, were visualized capturing and ingesting microbeads via their dendrites. Motile airwayadjacent DCs bearing beads were visualised 24 hours after bead inhalation. Further analysis showed that beads that were first taken up by alveolar DCs accumulated in the allergen-challenged airways over time. Of note, DC trafficking to the lymph node was not altered by the retention of microbead-bearing DCs within the airway-adjacent regions.

Finally, the authors assessed whether these retained DCs interact with antigen-specific T cells. They found that antigen-specific $T$ cells accumulated near the airways in response to allergen challenge and interacted with airway-adjacent DCs, moving from one DC to another. Furthermore, these interactions resulted in $\mathrm{T}$ cell activation, as determined by $\mathrm{Ca}^{2+}$ flux. By contrast, few $T$ cells were present in the alveolar regions, and they were rarely in contact with DCs.

So, these data suggest that antigen surveillance and presentation are regionally segregated in the allergic lung. The accumulation of antigen-bearing DCs and subsequent activation of antigen-specific $T$ cells in the airway-adjacent regions may explain why asthma pathogenesis is focused around the airways.

Olive Leavy

ORIGINAL RESEARCH PAPER Thornton, E. E. et al. Spatiotemporally separated antigen uptake by alveolar dendritic cells and airway presentation to T cells in the lung.J. Exp. Med. 14 May 2012 (doi:10.1084/jem.20112667)

FURTHER READING Braciale, T. J., Sun, J. \& Kim, T. S. Regulating the adaptive immune response to respiratory virus infection. Nature Rev. Immunol. 12, 295-305 (2012) 